\title{
CURVATURE AND TORSION FORMULAS FOR CONFLICT SETS
}

\author{
MARTIJN van MANEN \\ Mathematisch Instituut, Universiteit Utrecht \\ P.O.Box 80010, 3508 TA Utrecht, The Netherlands \\ E-mail:manen@math.uu.nl
}

\begin{abstract}
Conflict set are the points at equal distance from a number of manifolds. Known results on the differential geometry of these sets are generalized and extended.
\end{abstract}

Introduction. This paper is concerned with the study of curvature and regularity properties of conflict sets. Conflict sets are sets that are at equal distance from a number of hypersurfaces. The term "conflict set" was introduced by Thom. Conflict sets were studied in the context of curves in $\mathbb{R}^{2}$ in [Sie99]. There a simple formula for the curvature of the conflict set of two curves $\gamma_{i}$ with normal $n_{i}$ and curvature $\kappa_{i}$ was obtained.

This formula can be obtained in another way. Take two circles with centers $B_{1}$ and $B_{2}$. The line at equal distance from the two circles is a conic $C$. Its parametrization can be calculated and thus its curvature. If we denote by $T_{i}$ the signed distance from the center $B_{i}$ to a point $q$ on $C$, then the curvature of the hyperbola at $q$ is

$$
\kappa_{c}=\frac{1}{2}\left(\frac{1}{T_{1}}-\frac{1}{T_{2}}\right) \sin \alpha .
$$

Here $\alpha$ is half of the angle between $n_{1}$ and $n_{2}$, or what amounts to the same, the angle between $n_{i}$ and the tangent to the conflict set.

The quantities $\frac{1}{T_{i}}$ are the curvatures $\kappa_{i}$ of the curves propagated along the normals to the point $q$. One can argue with "contact-type" arguments that the case of two circles is representative for all other situations: essentially the $k$-jet of the conflict set is completely fixed once the $k$-jets of the $\gamma_{i}$ are known.

Several questions now arise:

- If the hypersurfaces are not smooth boundaries of mutually disjoint convex domains what then is the right definition of the conflict set? (Definition 1.3)

2000 Mathematics Subject Classification: Primary 53A07; Secondary 53B20.

The paper is in final form and no version of it will be published elsewhere. 


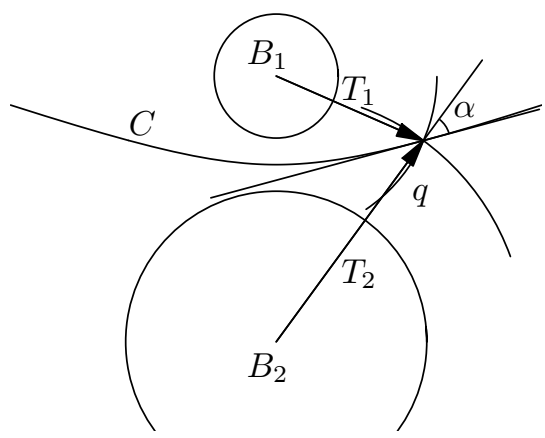

Figure 1. Illustration of formula (1)

- Once equipped with a good definition of a conflict set what are regular points of the conflict set? How can we assure smoothness? (Lemma 1.6)

- Does the $k$-jet of the conflict set depend only on the $k$-jets of the base manifolds? ("Jet theorem" 2.4)

- At regular points what is the curvature of the conflict set? (Theorems 2.5 and 3.1)

- If it the conflict set is a space curve what is the torsion? (Theorem 4.1)

We will provide reasonably complete answers to all of these questions. More details will be in $[\mathrm{vM}]$.

The referee points out that a series of interesting recent preprints by J. Damon deal with related questions. They are available on the Internet.

1. Regular points on a conflict set. Let a hypersurface $M$ be embedded in $\mathbb{R}^{n}$ by a $\mathcal{C}^{\infty}$ map $\gamma: M \rightarrow \mathbb{R}^{n}$. Denote by $n(s)$ the unit normal vector field to $M$. Consider the squared distance

$$
F(x, s)=\|x-\gamma(s)\|^{2}
$$

For $s_{0} \in M$ the normal $n$ defines a straight line with at each point of it a well-defined outward unit direction. If $p$ lies on a normal of $\gamma\left(s_{0}\right)$ we want to extend this direction to a well defined germ of a vector field in a neighborhood near $p \in \mathbb{R}^{n}$. At $\gamma\left(s_{0}\right)$ we have such a normal vector field, by the local diffeomorphism from $N M$ to $\mathbb{R}^{n}$. We assume that

$$
\operatorname{det} \frac{\partial^{2} F}{\partial s^{2}}\left(p, s_{0}\right) \neq 0
$$

This means that we are not on the focal sets, see [Mil63]. Consider the map

$$
\begin{aligned}
&(s, d) \stackrel{\Psi}{\rightarrow} \gamma(s)+d n, \\
& \Psi: N^{*} M \rightarrow \mathbb{R}^{n}
\end{aligned}
$$

It is a germ of a diffeomorphism from a neighborhood of $\left(\gamma\left(s_{0}\right),\|x-p\|\right)$ to a neighborhood of $p \in \mathbb{R}^{n}$. The push forward of the normal vector field near $\gamma\left(s_{0}\right)$ by $\Psi_{*}$ makes that, near $p$, the direction $n$ can be extended to a unit vector field. The situation is exemplified in Figure 2. 


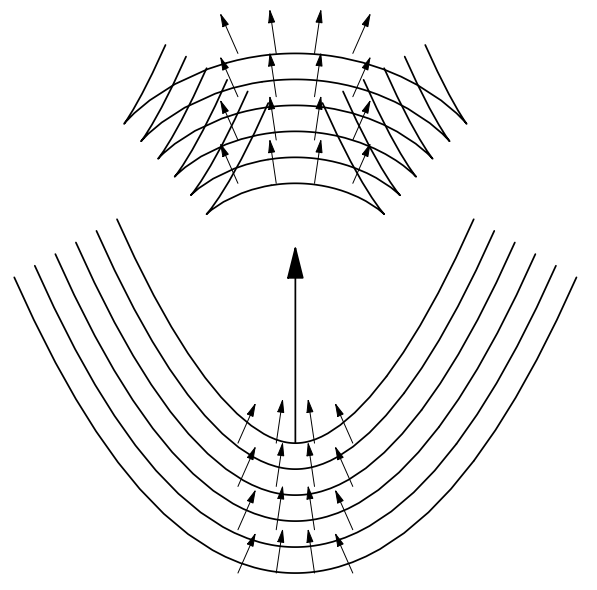

Figure 2. Push forward of normal vector field

Definition 1.1. If for the pair $\left(p, s_{0}\right) \in \mathbb{R}^{n} \times M$

- $p$ lies on the normal from $\gamma\left(s_{0}\right)$,

- there are no other points on $M$ whose normal contains $p$ and at the same distance from $p$ as $\gamma\left(s_{0}\right)$,

- the inequality (2) holds,

then we call $s_{0}$ or $\gamma\left(s_{0}\right)$ the base point of $p$ on $M$ at distance $\left\|x-\gamma\left(s_{0}\right)\right\|^{1 / 2}$.

ExAmple 1.2. If $M$ is the boundary of a strictly convex domain $A$, then any point outside $A$ has a unique base point at minimal distance on $M$.

DeFinition 1.3. Let us have $l$ hypersurfaces $M_{i}$ smoothly embedded in $\mathbb{R}^{n}$ by embeddings $\gamma_{i}: M_{i} \rightarrow \mathbb{R}^{n}$. The conflict set is the set of solutions to the equations

$$
F_{i}\left(x, s_{i}\right) \stackrel{\text { def }}{=}\left\|x-\gamma_{i}\left(s_{i}\right)\right\|, \quad \begin{cases}F_{i}\left(x, s_{i}\right)=F_{j}\left(x, s_{j}\right), & 1 \leq i<j \leq l \\ \frac{\partial F_{i}}{\partial s_{i}}=0, & 1 \leq i \leq l\end{cases}
$$

projected to $\mathbb{R}^{n}$.

REMARK 1.4. The equations (3) are not the only way to define a conflict set. There are at least two alternative definitions. First of all we could take graphs of distance functions and intersect them. Another way would be to take the closure of the points where the distance functions to each of the manifolds have a simple minimum at the same level.

REMARK 1.5. We restrict our attention to hypersurfaces. This is mainly because the equidistant of a codimension greater than 1 submanifold is no longer a codimension greater than 1 manifold but a hypersurface.

LEMMA 1.6. If $p$ lies on the conflict set and

- $p$ has base points on each of the $M_{i}$,

- the normals $n_{i}$ lie affinely in general position, i.e. the differences $n_{1}-n_{i}$ are linearly independent, then the conflict set is a germ of an immersed manifold near $p$. 
Proof. The partial derivative matrix of the set of $l-1+l(n-1)$ equations (3) should have maximal rank. The matrix is

$$
K=\left(\begin{array}{cccccc}
\frac{\partial F_{1}}{\partial x}-\frac{\partial F_{2}}{\partial x} & 0 & \ldots & \ldots \ldots & \ldots & 0 \\
\ldots \ldots \ldots \ldots & \ldots & \ldots & \ldots \ldots & \ldots & \ldots \\
\frac{\partial F_{l}-1}{\partial x}-\frac{\partial F_{l}}{\partial x} & 0 & \ldots & \ldots \ldots & \ldots & 0 \\
\frac{\partial^{2} F_{1}}{\partial x \partial s_{1}} & \frac{\partial^{2} F_{1}}{\partial s_{1}^{2}} & 0 & \ldots \ldots & \ldots & 0 \\
\ldots \ldots \ldots & \ldots & \ldots & \ldots \ldots & \ldots & \ldots \\
\frac{\partial^{2} F_{l}}{\partial x \partial s_{l}} & 0 & \ldots & \ldots \ldots & 0 & \frac{\partial^{2} F_{l}}{\partial s_{l}^{2}}
\end{array}\right)
$$

The $n_{i}$ are unit vectors of $\frac{\partial F_{i}}{\partial x}$. Because the $n_{i}$ are affinely in general position, the upper rows of the matrix $K$ are linearly independent. Because we are not on focal sets the others are so too. Next we need to project to the $x$-space. The lower right $l(n-1) \times l(n-1)$ square of the matrix $K$ has maximal rank and this shows that the projection is also non-singular.

The conditions in the lemma above do not preclude all unwanted situations: immersed manifolds may not really be manifolds. There can be self-intersections, other components can be arbitrary close, etc. We want the conflict set to really be a manifold. Many conditions can ensure this, but as we only consider local situations we do not delve deeper into these matters.

DEFInITION 1.7. If the conditions of the previous lemma are satisfied and also the conflict set is an embedded sub-manifold at $q$ then we call $q$ a regular point of the conflict set.

We will employ the notation

- $M_{c}=C\left(M_{1}, \ldots, M_{l}\right)$ for the conflict set of the $M_{i}$.

- $n_{i}$ for the normal vector field of $M_{i}$ transported to $p$.

- $p$ for a regular point on the conflict set with base points $p_{i}$.

Throughout this article all points on the conflict set that we consider are regular.

For the local part of the conflict set we study we can and will assume that $p_{i}=p$ : the patches of the wavefronts at $p$ determine the same patch of a conflict set.

Now we are ready to describe the tangent space to the conflict set. The tangent planes to the base points are hyperplanes $V_{i}$ in $\mathbb{R}^{n}$.

LEMMA 1.8. The tangent space of the conflict set is the tangent space to the conflict set of the tangent planes at the base points.

Proof. It will be enough to prove this for two hypersurfaces. By coordinate changes the two hypersurfaces can be made to look like

$$
x_{n}=a_{i} x_{1}+Q_{i}\left(x_{1}, \ldots, x_{n-1}\right), \quad i=1,2,
$$

where $a_{1}=-a_{2}$, and the $Q_{i}$ contain no linear terms. The implicit function theorem concludes the proof.

Now we easily calculate the tangent space to the conflict set. It is just the set of vectors orthogonal to each of the differences $n_{i}-n_{i+1}$. If $n=l$ then there is only one 
such direction, it is the sum of cross products of

$$
t_{c}=\left(n_{1}-n_{2}\right) \times\left(n_{2}-n_{3}\right) \times \ldots \times\left(n_{l-1}-n_{l}\right)=\sum_{i=1}^{l}(-1)^{(i+1)(l+1)}{\underset{j=1}{l-1} \times}_{n_{q(i, j, l}},
$$

where $q(i, j, l)=1+((i+j-1) \bmod l)$. The function $q$ is a "circulator" over a finite index set $\{1, \ldots, l\}$. So $q(1,1,4)=2, q(1,2,4)=3, q(1,3,4)=4$, and $q(1,4,4)=1$.

If $n=3$ this reduces to

$$
t_{c}=n_{1} \times n_{2}+n_{2} \times n_{3}+n_{3} \times n_{1} .
$$

If $n=l$ then the conflict set is a space curve in $\mathbb{R}^{n}$. According to (4) the unit tangent to the conflict set is

$$
e=\frac{t_{c}}{\left|t_{c}\right|}
$$

The tangent to the conflict set is defined in terms of the $n_{i}$ so $e$ is a unit vector field in a neighborhood of $p$. The part of $e$ orthogonal to $n_{i}$ is also a vector field near $p$.

$$
t_{i}^{\prime}=e-\left\langle e, n_{i}\right\rangle n_{i} \quad \text { and } \quad t_{i}=\frac{t_{i}^{\prime}}{\left|t_{i}^{\prime}\right|} .
$$

The integral curve from $p$ is a germ of a curve in $M_{i}$. We shall call it the base curve. It is the curve traced out by the base point on $M_{i}$. The base curve in $M_{i}$ has normal curvature $\kappa_{i}$. The normal curvature depends only on the second order Taylor series of the embedding of $M_{i}$ at $p$ and the direction $t_{i}$. The normal curvature has a derivative $D_{t_{i}} \kappa_{i}$ that depends only on the third order Taylor series of the embedding of $M_{i}$ at $p$. We will encounter $D_{t_{i}} \kappa_{i}$ in the section where we calculate the torsion of the conflict set in the case $n=l=3$.

2. Contact and a jet theorem. Two germs of submanifolds $M$ and $N$ can have different sorts of contact at a point $p \in M \cap N$, there can be a transversal intersection, tangent space can coincide, etc. Denote by $\sigma_{M}$ and $\iota_{M}$ germs of a submersion and immersion defining $M$ at $p$. The contact between $M$ and $N$ is measured with the contact class, described in [GG73]. It is the equivalence class under $V$-equivalence of $\sigma_{M} \circ \iota_{N}$.

If $M$ is $m$-dimensional and $N$ is $q$-dimensional then $\kappa_{M, N}=\sigma_{M} \circ \iota_{N}: \mathbb{R}^{q} \rightarrow \mathbb{R}^{n-m}$ while $\kappa_{N, M}=\sigma_{N} \circ \iota_{M}: \mathbb{R}^{m} \rightarrow \mathbb{R}^{n-q}$. If $q=m$ then these maps are contact-equivalent. If $q<m$ then $\kappa_{N, M}$ is contact-equivalent to $\kappa_{M, N} \times \operatorname{Id}_{m-q}$. With $\operatorname{Id}_{m-q}$ meaning the identity map on $\mathbb{R}^{m-q}$. For details and proofs see [Mon86].

The $k$-th derivative of $\kappa_{N, M}$ is a symmetric $\mathbb{R}^{n-q}$-valued $k$-form on $T_{p} M$. We can view it as an $\mathbb{R}^{n-q}$-valued $k$-form on $\left(T_{p} M \cap T_{p} N\right) \subset T_{p} \mathbb{R}^{n}$ by the push forward $\left(\iota_{M}\right)_{*}$ : $T_{p} M \rightarrow T_{p} \mathbb{R}^{n}$

Definition 2.1. If on a linear subspace $V \subset T_{p} M \cap T_{p} N \subset T_{p} \mathbb{R}^{n}$ the forms $\mathrm{d}^{i} \kappa_{N, M}$ annul for each $i$ not exceeding $k$ then we say that $M$ and $N$ have a $(k+1)$-contact in the directions $V$ at $p$.

We have to check that this is well-defined. It is clear that the definition does not depend on the choice of immersion for $M$ or submersion for $N$. Next we need to show 
that

$$
\left(\forall i \leq\left. k \mathrm{~d}^{i} \kappa_{M, N}\right|_{V} \equiv 0\right) \Longrightarrow\left(\forall i \leq\left. k \mathrm{~d}^{i} \kappa_{N, M}\right|_{V} \equiv 0\right)
$$

(where the derivatives are to be evaluated in $p$ ). This follows from the contact-equivalence. Let

$$
\lambda_{M, N}=\kappa_{M, N} \times \operatorname{Id}_{m-q} .
$$

These maps are contact-equivalent. According to Proposition 2.3 in [Mat68] we have

$$
\lambda_{M, N}=A(x)\left(\kappa_{N, M} \circ g\right)
$$

and $A: \mathbb{R} \rightarrow \operatorname{GL}(q)$. Because $A \circ \sigma_{N}$ is also a submersion for $N$ and $\iota_{M} \circ g$ also an immersion for $M$ at $p$. Hence we can assume $\lambda_{M, N}=\kappa_{N, M}$. Thus this notion of contact is well-defined. Of course it is cruder than the contact class.

EXAMPLE 2.2.

- If $M$ and $N$ have a 2-contact along $T_{p} N$ then $T_{p} N \subset T_{p} M$.

- If $T_{p} M=T_{p} N$ for two hypersurfaces in $\mathbb{R}^{n}$ and they have a 3-contact in the direction $v \in T_{p} \mathbb{R}^{n}$ then the normal curvatures in that direction $v$ of these hypersurfaces are equal.

- If

$$
\mathbb{R}^{n-1} \ni t \rightarrow\left(t, \frac{1}{2}\left\langle B_{i} t, t\right\rangle\right), \quad i=1,2,
$$

are two Monge forms for a hypersurface in $\mathbb{R}^{n}$ their contact map has second derivative $B_{1}-B_{2}$.

As can be seen from these examples, the contact between sub-manifolds is a good measure for how close they are. The next lemma formalizes our last example.

LEMma 2.3. Consider a submanifold $L$ of $\mathbb{R}^{n}$, two hypersurfaces $M$ and $N$. Let $T_{p} L \subset T_{p} M=T_{p} N$ and fix an immersion for $L$ at $p$. If $j^{k} \kappa_{N, L}=j^{k} \kappa_{M, L}$ then $M$ and $N$ have a $(k+1)$-contact along $T_{p} L$.

Proof. We may assume that $\iota_{L}: \mathbb{R}^{k} \rightarrow \mathbb{R}^{n}$ is $t^{\prime} \mapsto\left(t^{\prime}, 0\right) . M$ has an immersion $\left(t^{\prime}, t^{\prime \prime}\right) \mapsto\left(t^{\prime}, t^{\prime \prime}, g_{M}\left(t^{\prime}, t^{\prime \prime}\right)\right)$. In this way a submersion for $N\left(=x_{n}-g_{N}\left(x_{1}, \ldots, x_{n-1}\right)\right)$ can be chosen as well. With these choices the lemma is easily proven.

We have introduced this notion of contact so that we can compare conflict sets $M_{c}=$ $C\left(M_{1}, \ldots, M_{l}\right)$ and $N_{c}=C\left(N_{1}, \ldots, N_{l}\right)$. For 2-contact we have already done this in Lemma 1.8. Something more general is true.

Theorem 2.4 (Jet theorem). Assume that $M_{i}$ and $N_{i}$ have a 2 -contact at $p_{i}$. Let the hypersurfaces $M_{i}$ and $N_{i}$ in addition have a $k$-contact in the directions $V_{i} \subset T_{p_{i}} M_{i}=$ $T_{p_{i}} N_{i}$. Suppose $V \subset T_{p} M_{c}$ is such that the projection $\pi_{i}$ from $T_{p} M_{c}$ to each of the $T_{p} M_{i}$ maps $V$ to $V_{i}$.

Then the conflict sets $N_{c}$ and $M_{c}$ have a $k$-contact in the directions $V$.

As an application of this theorem we get the curvature formula (1) in general, not just for two circles. Indeed with two curves we can find two circles that have a 3-contact with the curves at the base points. They are the osculating circles. The theorem says that the 
conflict set of the curves has a 3-contact with the conflict set of the two circles and thus equal curvature. In (1) we calculate the curvature of the conflict set of the two circles. And thus formula (1) holds in general.
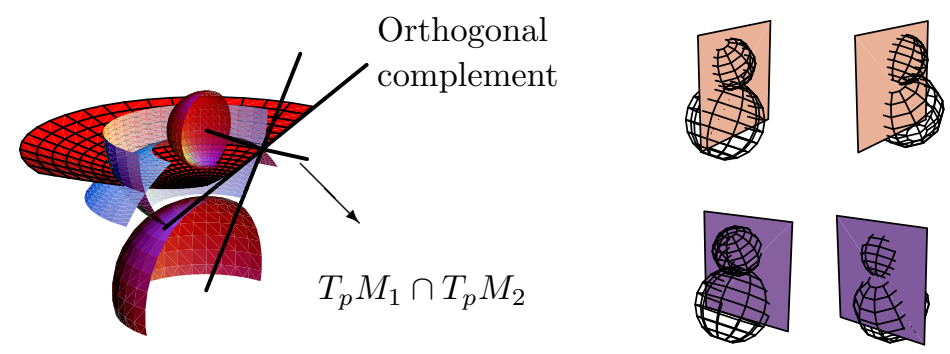

Figure 3. Two orthogonal directions in $T_{p} M_{c}$ account for two different curvature formulas

Another application is the curvature formula for two hypersurfaces in $\mathbb{R}^{n}$. Here we apply formula (1) to each direction in $T_{p} M_{c}$. The tangent space to the conflict set naturally splits up in two parts. An $(n-2)$-dimensional part of the tangent space to the conflict set is common to $T_{p} M_{1}, T_{p} M_{2}$ and $T_{p} M_{c}$. In that case we have to look at the right upper corner of Figure 3. Thus for these directions we have simple formula to find the curvature. We have from the right lower corner of Figure 3

$$
\kappa_{c}=\frac{1}{2}\left(\frac{\kappa_{1}}{\sin \alpha}-\frac{\kappa_{2}}{\sin \alpha}\right) \text {. }
$$

In the direction that is the orthogonal complement of $T_{p} M_{c}$ in $T_{p} M_{i}$ the curvature is simply given by the formula (1):

$$
\kappa_{c}=\frac{1}{2}\left(\kappa_{1}-\kappa_{2}\right) \sin \alpha .
$$

We see this from the right lower corner of Figure 3. Combining this and writing the projection matrix $\mathbf{P}: T_{p} M_{c} \rightarrow T_{p} M_{i}$ as

$$
\mathbf{P}=\left(\begin{array}{ccccc}
1 & 0 & \ldots & \ldots & 0 \\
0 & 1 & \ldots & \ldots & 0 \\
\ldots & \ldots & \ldots & \ldots & \ldots \\
0 & \ldots & \ldots & 1 & 0 \\
0 & \ldots & \ldots & 0 & \sin \alpha
\end{array}\right)
$$

we obtain from the jet theorem an $n$-dimensional analogue of the formula of Siersma [Sie99] and a generalization of the results in [SSG99].

THEOREM 2.5. Denote by $\mathbf{I}_{i}$ the second fundamental form of the equidistant of $M_{i}$ at $p$. The second fundamental form of the conflict set is

$$
\mathbf{I}_{c}=\frac{1}{2 \sin \alpha} \mathbf{P}^{T}\left(\mathbf{I}_{1}-\mathbf{I}_{2}\right) \mathbf{P} .
$$


Proof of the jet theorem. In the definition of the conflict set we can write instead of $F_{i}\left(x, s_{i}\right)=F_{j}\left(x, s_{j}\right)$

$$
d=F\left(x, s_{i}\right) \text { or } d=\left\|x-\gamma\left(s_{i}\right)\right\|, \quad 1 \leq i \leq l .
$$

Writing this we exhibit the conflict set as an intersection of a number of hypersurfaces in $\mathbb{R}^{n+1}$, graphs of the distance functions to the $M_{i}$. So another way to obtain the conflict set is to intersect those and project the intersection to $\mathbb{R}^{n}$.

At regular points in the sense of Definition 1.7 this intersection is transversal. We need a lemma on contact and transversal intersections.

LEMMA 2.6. If

- $N_{i}$ and $M_{i}$ have a $k$-contact along the directions $V_{i} \subset T_{p} N_{i} \cap T_{p} M_{i}$,

- both $\bigcap_{i=1}^{l} M_{i}$ and $\bigcap_{i=1}^{l} N_{i}$ intersect transversally,

then $\bigcap_{i=1}^{l} M_{i}$ and $\bigcap_{i=1}^{l} N_{i}$ have a $k$-contact along $\bigcap_{i=1}^{l} V_{i}$.

Proof. To show the claim we combine two remarks.

First, the $(k+1)$-st derivative of the contact-map $\kappa_{\cap N, \cap M}$ is just the restriction to $T_{p}(\bigcap M)$ of $\kappa_{\bigcap N, M_{i}}$. Thus each of the $N_{i}$ has a $k$-contact with the $\bigcap_{i=1}^{l} M_{i}$ in the directions $\bigcap_{i=1}^{l} V_{i}$.

Second, a submersion for $\bigcap_{i=1}^{l} N_{i}$ is simply

$$
\sigma_{\cap N}=\left(\sigma_{N_{1}}, \ldots, \sigma_{N_{l}}\right): \mathbb{R}^{n} \rightarrow \mathbb{R}^{\sum \operatorname{codim} N_{i}} .
$$

We also need a lemma on equidistants and contact.

LEMMA 2.7. Let hypersurfaces $M_{1}$ and $M_{2}$ have a 2-contact and a $k$-contact in some directions $V$ at $q \in \mathbb{R}^{n}$. If we are away from focal sets, that is inequality (2) holds, then their equidistants have the same contact at $p$.

Proof. Immersions for $M_{i}$ can be written as

$$
\mathbb{R}^{n-1} \ni t \rightarrow\left(t, g_{i}(t)\right), \quad i=1,2 \text { such that } \frac{\partial g_{i}}{\partial t}=0 .
$$

We repeatedly apply Lemma 2.3. By hypothesis the $(k-1)$-jets of $g_{1}$ and $g_{2}$ agree at 0 . If $n_{i}: \mathbb{R}^{n-1} \rightarrow \mathbb{R}^{n}$ is the normal to $M_{i}$, then the $(k-2)$-jets of $n_{1}$ and $n_{2}$ agree. At $\left(d_{0}, p\right) \in \mathbb{R}^{n+1}$ the patches defined by $(d, t) \rightarrow(d, \gamma(t)+d \cdot n(t))$ thus have a $(k+1)$ contact along directions spanned by $V$ and $n_{i}$. Intersect with a plane $d=$ constant to obtain the conclusion.

From the proof of the above lemma the hypersurfaces defined by

$$
t=F_{i}\left(x, \gamma_{i}\left(s_{i}\right)\right), \quad \frac{\partial F_{i}}{\partial s_{i}}=0,
$$

clearly have a $k$-contact in the direction

$$
\left\{\left(\lambda_{1} v, 0\right)+\lambda_{2}\left(n_{i}, 1\right) \in T_{p} \mathbb{R}^{n+1} \mid v \in V, \lambda_{i} \in \mathbb{R}\right\}
$$

if $V$ is the direction along which the surfaces $M$ and $N$ have a $k$-contact.

If $M_{i}$ and $N_{i}$ are like in the statement of the theorem we first take their equidistants equid $M_{i}$ and equid $N_{i}$. They have the same contact as $M_{i}$ and $N_{i}$. Then we construct the 
hypersurfaces (8). They have contact in the directions spanned by $V_{i}$ and $n_{i}$. Intersect those and project to $\mathbb{R}^{n}$.

3. Curvature formulas. We have insisted that the $n_{i}$ are defined as vector fields. The benefit is that we can use covariant differentiation to obtain a curvature formula for the conflict set of three surfaces. In this way we avoid the use of coordinates.

TheOREM 3.1. Let $\kappa_{i}$ be the normal curvature along the base curve at $p$. Let $\alpha$ be the angle between e and $n_{i}$. The curvature of the conflict set of three hypersurfaces in $\mathbb{R}^{3}$ is

$$
\kappa_{c}^{2}=\sin ^{6} \alpha \sum_{i=1}^{3} \frac{\left(\kappa_{i}-\kappa_{q(i, 1,3)}\right)\left(\kappa_{q(i, 1,3)}-\kappa_{q(i, 1,3)}\right)}{\left(1-\left\langle n_{i}, n_{q(i, 1,3)}\right\rangle\right)\left(\left\langle n_{q(i, 1,3)}, n_{q(i, 2,3)}\right\rangle-1\right)} .
$$

A normal to the conflict set is

$$
n_{c}=\kappa_{1} e \times\left(n_{2}-n_{3}\right)+\kappa_{2} e \times\left(n_{3}-n_{1}\right)+\kappa_{3} e \times\left(n_{1}-n_{2}\right) .
$$

This curvature is zero if and only if all three curvatures $\kappa_{i}$ are equal.

Proof. The absolute value of the curvature of a space curve with unit tangent $e$ is the length of the vector $D_{e} e$. By the extension of the normals to normal vector fields near $p \in \mathbb{R}^{n}$ we can calculate $D_{e} e$ as the covariant derivative of a vector field.

$$
D_{e} e=D_{e} \frac{t_{c}}{\left|t_{c}\right|}=\frac{\left|t_{c}\right|^{2} D_{e} t_{c}-\left\langle t_{c}, D_{e} t_{c}\right\rangle t_{c}}{\left|t_{c}\right|^{3}} .
$$

From the Frenet-Serret equations we know that $\left\langle D_{e} e, e\right\rangle=0$. Choose coordinates so that at $p$

$$
e=\left(\begin{array}{l}
0 \\
0 \\
1
\end{array}\right), \quad n_{i}=\left(\begin{array}{c}
\sin \alpha \sin \beta_{i} \\
\sin \alpha \cos \beta_{i} \\
\cos \alpha
\end{array}\right), \quad t_{i}=\left(\begin{array}{c}
-\cos \alpha \sin \beta_{i} \\
-\cos \alpha \cos \beta_{i} \\
\sin \alpha
\end{array}\right)
$$

Then if we want to determine the value of $D_{e} e$ at $p$ all we need to determine are the first and second components. Thus according to (10) we need to determine the first and second components of

$$
\begin{aligned}
\frac{D_{e} t_{c}}{\left|t_{c}\right|}= & \frac{D_{e}\left(n_{1} \times n_{2}+n_{2} \times n_{3}+n_{3} \times n_{1}\right)}{\left|t_{c}\right|} \\
& =\frac{D_{e} n_{1} \times\left(n_{2}-n_{3}\right)+D_{e} n_{2} \times\left(n_{3}-n_{1}\right)+D_{e} n_{3} \times\left(n_{1}-n_{2}\right)}{\left|t_{c}\right|} .
\end{aligned}
$$

The quantities to calculate are

$$
D_{e} n_{1} \times\left(n_{2}-n_{3}\right), \quad D_{e} n_{2} \times\left(n_{3}-n_{1}\right), \quad D_{e} n_{3} \times\left(n_{1}-n_{2}\right), \quad\left|t_{c}\right| .
$$

Because

$$
e=\cos \alpha n_{i}+\sin \alpha t_{i}
$$

at $p$ and obviously $D_{n_{i}} n_{i}=0$ we have at $p$

$$
D_{e} n_{i}=\sin \alpha D_{t_{i}} n_{i}=\sin \alpha \kappa_{i} t_{i} .
$$


Using the coordinates from (11) we obtain

$$
D_{e} n_{1} \times\left(n_{2}-n_{3}\right)=\kappa_{1} \sin ^{3} \alpha\left(\begin{array}{c}
\sin \beta_{2}-\sin \beta_{3} \\
\cos \beta_{3}-\cos \beta_{2} \\
\ldots
\end{array}\right)
$$

(In view of $e=(0,0,1)$ equation (9) is now proven.) Consequently the squared sum of the first and second components of the numerator of (12) is:

$$
\begin{gathered}
-4 \sin ^{6} \alpha\left(\left(\kappa_{1}-\kappa_{2}\right)\left(\kappa_{2}-\kappa_{3}\right) \sin ^{2}\left(\frac{\beta_{3}-\beta_{1}}{2}\right)+\left(\kappa_{2}-\kappa_{3}\right)\left(\kappa_{3}-\kappa_{1}\right) \sin ^{2}\left(\frac{\beta_{1}-\beta_{2}}{2}\right)\right. \\
\left.\quad+\left(\kappa_{3}-\kappa_{1}\right)\left(\kappa_{1}-\kappa_{2}\right) \sin ^{2}\left(\frac{\beta_{2}-\beta_{3}}{2}\right)\right) \\
=-4 \sin ^{6} \alpha \sum_{i=1}^{3} \sin ^{2}\left(\frac{\beta_{q(i, 1,3)}-\beta_{q(i, 2,3)}}{2}\right)\left(\kappa_{q(i, 2,3)}-\kappa_{i}\right)\left(\kappa_{i}-\kappa_{q(i, 1,3)}\right) .
\end{gathered}
$$

In the coordinates (11) the expression for $t_{c}$ reads:

$$
\left|t_{c}\right|^{2}=16 \sin ^{4} \alpha \sin ^{2}\left(\frac{\beta_{1}-\beta_{2}}{2}\right) \sin ^{2}\left(\frac{\beta_{2}-\beta_{3}}{2}\right) \sin ^{2}\left(\frac{\beta_{3}-\beta_{1}}{2}\right) .
$$

In these formulas we can replace the $\sin \left(\beta_{i} / 2-\beta_{q(i, 1, l)} / 2\right)$ by a somewhat simpler expression:

$$
1-\left\langle n_{1}, n_{2}\right\rangle=2 \sin ^{2} \alpha \sin ^{2}\left(\frac{\beta_{1}-\beta_{2}}{2}\right) .
$$

Inserting this into (13) and (14) concludes the proof.

In the case of three spheres $\kappa_{c}=0$ iff the three curvatures are equal, see Section 5 below. The jet theorem now concludes the proof.

4. Torsion formulas. With three disjoint spheres the conflict set is a plane curve. In general of course the conflict set may have torsion. The expression for the torsion that we derive is dependent on the curvature $\kappa_{c}$. With notations explained in the remarks below we have the following theorem and formula:

THEOREM 4.1. If $\kappa_{c} \neq 0$, the torsion $\tau_{c}$ of the conflict set is

$$
\tau_{c}= \pm \frac{\sin ^{5} \alpha}{\left|t_{c}\right| \kappa_{c}^{2}}\left(\kappa_{1}\left(D_{t_{2}} \kappa_{2}^{\prime}-D_{t_{3}} \kappa_{3}^{\prime}\right)+\kappa_{2}\left(D_{t_{3}} \kappa_{3}^{\prime}-D_{t_{1}} \kappa_{1}^{\prime}\right)+\kappa_{3}\left(D_{t_{1}} \kappa_{1}^{\prime}-D_{t_{2}} \kappa_{2}^{\prime}\right)\right) .
$$

REMARK 4.2. Compare this formula to a classical formula for the torsion of a space curve with nonzero curvature $\kappa$ and a unit parametrization $c(t)$ (see [Spi])

$$
\tau=\frac{1}{\kappa^{2}}\left\langle\frac{\mathrm{d} c}{\mathrm{~d} t} \times \frac{\mathrm{d}^{2} c}{\mathrm{~d} t^{2}}, \frac{\mathrm{d}^{3} c}{\mathrm{~d} t^{3}}\right\rangle .
$$

We see that the term $\frac{1}{\kappa_{c}^{2}}$ is natural in this respect.

REMARK 4.3. The meaning of the term $D_{t_{i}} \kappa_{i}^{\prime}$ is as follows. It is the derivative of the normal curvature along the base curve on the equidistant at $p$. The relation between normal curvature and normal curvature on an equidistant is clear. At distance $d$ the 
normal curvature is

$$
\frac{\kappa}{1+d \kappa}
$$

Where their derivatives are concerned this is much less the case. Let us start by clarifying how the 3-jet of a hypersurface determines the derivative of the normal curvature.

In case of an immersion that up to a quadratic form looks like

$$
(s, t) \mapsto\left(s, t, \frac{1}{6}\left(A s^{3}+3 B s^{2} t+3 C s t^{2}+D t^{3}\right)\right)
$$

and a curve with tangent $(\cos \mu, \sin \mu)$ the derivative is

$$
A \cos ^{3} \mu+3 B \cos ^{2} \mu \sin \mu+3 C \cos \mu \sin ^{2} \mu+D \sin ^{3} \mu .
$$

So that - as was to be expected - if two surfaces have 2-contact and 4-contact in the direction $\vec{v}$ curves in that direction on the surfaces have equal derivatives of the normal curvature.

The relation between the derivative of the normal curvature at the base manifold and at its equidistant is most conveniently found by just using curves in $\mathbb{R}^{2}$. If we want a curve with prescribed derivative of the curvature $\alpha$ at 0 we can take

$$
\gamma: t \mapsto\left(t, \frac{\kappa}{2} t^{2}+\frac{\alpha}{6} t^{3}\right)
$$

At distance $d$ its derivative of the normal curvature is

$$
\frac{\alpha}{(1+d \kappa)^{3}} \text {. }
$$

Proof of the torsion formula. To derive the torsion formula we need the expression for the principal normal to the conflict set, because we want to apply the Frenet-Serret equations.

If $\kappa_{c} \neq 0$ then at least two of the $\kappa_{i}$ are different, for instance $\kappa_{1} \neq \kappa_{2}$. Thus we can use (9) for the normal to the conflict set. The binormal is $e \times n_{c}$. With

$$
e \times\left(e \times\left(n_{1}-n_{2}\right)\right)=n_{2}-n_{1}
$$

we have

$$
b_{c}=\kappa_{1}\left(n_{2}-n_{3}\right)+\kappa_{2}\left(n_{3}-n_{1}\right)+\kappa_{3}\left(n_{1}-n_{2}\right) .
$$

Of course we will have $\left|b_{c}\right|=\left|n_{c}\right|$ and $\left\langle n_{c}, b_{c}\right\rangle=0$. The Frenet-Serret equations say that

$$
D_{e} \frac{b_{c}}{\left|b_{c}\right|}=\frac{\left|b_{c}\right|^{2} D_{e} b_{c}-\left\langle b_{c}, D_{e} b_{c}\right\rangle b_{c}}{\left|b_{c}\right|^{3}}=-\tau_{c} \frac{n_{c}}{\left|n_{c}\right|}
$$

whence we have

$$
\tau_{c}=-\frac{\left\langle D_{e} b_{c}, n_{c}\right\rangle}{\left|b_{c}\right|^{2}} .
$$

We need to determine $D_{e} b_{c}$ and $\left|b_{c}\right|^{2}$. For $\left|b_{c}\right|^{2}$ we can use the calculation from the previous theorem:

$$
-4 \sin ^{2} \alpha \sum_{i=1}^{3} \sin ^{2}\left(\frac{\beta_{1}-\beta_{q(i, 1,3)}}{2}\right)\left(\kappa_{q(i, 1,3)}-\kappa_{q(i, 2,3)}\right)\left(\kappa_{q(i, 2,3)}-\kappa_{i}\right)=\frac{\kappa_{c}^{2}\left|t_{c}\right|^{2}}{\sin ^{4} \alpha} .
$$


For $D_{e} b_{c}$ a little more work needs to be done. Inevitably we will stumble upon terms involving $D_{e} \kappa_{i}$. The normal curvature of the base manifold in the direction of the base curve naturally extends to a $\mathcal{C}^{\infty}$ function near $p \in \mathbb{R}^{n}$ by taking parallels to the base curve in $M_{i}$ and then parallels to $M_{i}$. Thus the expression $D_{e} \kappa_{i}$ is meaningful.

To find $D_{e} \kappa_{i}$ split $e$ as before up in two parts: $e=n_{i} \cos \alpha+t_{i} \sin \alpha$. Then

$$
D_{e} \kappa_{i}=\cos \alpha D_{n_{i}} \kappa_{i}+\sin \alpha D_{t_{i}} \kappa_{i}^{\prime}
$$

Along the normal the curvature is

$$
\frac{1}{1 / \kappa_{i}+t}
$$

Clearly $D_{n_{i}} \kappa_{i}$ is found by differentiating (20) with respect to $d$, and evaluating at 0 . Thus

$$
D_{n_{i}} \kappa_{i}=\left.\frac{\partial}{\partial t}\left(\frac{1}{1 / \kappa_{i}+t}\right)\right|_{t=0}=-\frac{1}{\kappa_{i}^{2}} .
$$

The other expression is the derivative of the normal curvature along the base curve, its geometrical signification was explained in the above remarks.

All in all we have

$$
\begin{aligned}
D_{e} b_{c}= & \sin \alpha\left(\kappa_{1}\left(\kappa_{2} t_{2}-\kappa_{3} t_{3}\right)+\kappa_{2}\left(\kappa_{3} t_{3}-\kappa_{1} t_{1}\right)+\kappa_{3}\left(\kappa_{1} t_{1}-\kappa_{2} t_{2}\right)\right) \\
& -\cos \alpha\left(\kappa_{1}^{2}\left(n_{2}-n_{3}\right)+\kappa_{2}^{2}\left(n_{3}-n_{1}\right)+\kappa_{3}^{2}\left(n_{1}-n_{2}\right)\right) \\
& +\sin \alpha\left(D_{t_{1}} \kappa_{1}^{\prime}\left(n_{2}-n_{3}\right)+D_{t_{2}} \kappa_{2}^{\prime}\left(n_{3}-n_{1}\right)+D_{t_{3}} \kappa_{3}^{\prime}\left(n_{1}-n_{2}\right)\right) \\
= & I+I I+I I I .
\end{aligned}
$$

It can be shown that $\left\langle I+I I, n_{c}\right\rangle=0$.

We have

$$
\begin{aligned}
& \left\langle D_{e} b_{c}, n_{c}\right\rangle=\left\langle I I I, n_{c}\right\rangle \\
= & \sin \alpha\left|t_{c}\right|\left(\kappa_{1}\left(D_{t_{2}} \kappa_{2}^{\prime}-D_{t_{3}} \kappa_{3}^{\prime}\right)+\kappa_{2}\left(D_{t_{3}} \kappa_{3}^{\prime}-D_{t_{1}} \kappa_{1}^{\prime}\right)+\kappa_{3}\left(D_{t_{1}} \kappa_{1}^{\prime}-D_{t_{2}} \kappa_{2}^{\prime}\right)\right) .
\end{aligned}
$$

Combining (22) and (19) in (18) we have found the formula.

5. Example: three spheres in $\mathbb{R}^{3}$. Suppose we have three spheres $M_{i}$ in $\mathbb{R}^{3}$. Denote their centers by $p_{i}, i=1,2,3$, and their radii by $r_{i}$. It turns out that we can calculate an explicit parametrization of the conflict set of these three surfaces. Two elementary lemmas are the basis for such a calculation.

LEMMA 5.1. If the convex hull of the $\left\{p_{i}\right\}_{i=1,2,3}$ is an acute triangle then coordinates can be chosen so that mutual distances are preserved and $p_{1}=\left(b_{1}, 0,0\right), p_{2}=\left(0, b_{2}, 0\right)$, $p_{3}=\left(0,0, b_{3}\right)$.

Proof. Each pair $p_{i}, p_{j}$ can be used as the poles for a sphere with radius $\left|p_{i}-p_{j}\right|$. Thus there are three spheres. These intersect iff the triangle $\Delta\left(p_{1}, p_{2}, p_{3}\right)$ is acute angled. The intersection of the three spheres is the origin $O=(0,0,0)$. The angle $\angle p_{i} O p_{j}$ is an inscribed angle in a semi-circle, therefore it is right. Thus the three lines through $O$ and $p_{i}$ can serve as coordinate axes. 
LEMma 5.2. If $\left|p_{i}-p_{j}\right| \neq \pm r_{i} \pm r_{j}$ then the conflict set is a collection of conic sections.

Proof. Each part of the conflict set is given by two equations.

$$
\begin{aligned}
& \left|x-p_{2}\right|=\left|x-p_{1}\right|-a_{1}+a_{2} \\
& \left|x-p_{3}\right|=\left|x-p_{1}\right|-a_{1}+a_{3} .
\end{aligned}
$$

where the $a_{i}= \pm r_{i}$. If we square those and if $a_{1} \neq a_{2}$ we can find a plane in which the conflict set lies, by eliminating $\left|x-p_{1}\right|$. If $a_{1}-a_{2}= \pm\left|p_{1}-p_{2}\right|$ then the solution of the first of these equations is already a half-line. The corresponding conflict set can be empty. In all other cases we can find a plane in which this part of the conflict set lies. Whether this part is empty or not depends on the sign of

$$
\left(\left|p_{1}-p_{2}\right|^{2}-\left(a_{1}-a_{2}\right)^{2}\right)\left(\left|p_{2}-p_{3}\right|^{2}-\left(a_{2}-a_{3}\right)^{2}\right)\left(\left|p_{3}-p_{1}\right|^{2}-\left(a_{3}-a_{1}\right)^{2}\right),
$$

as one can show with a little computer algebra.

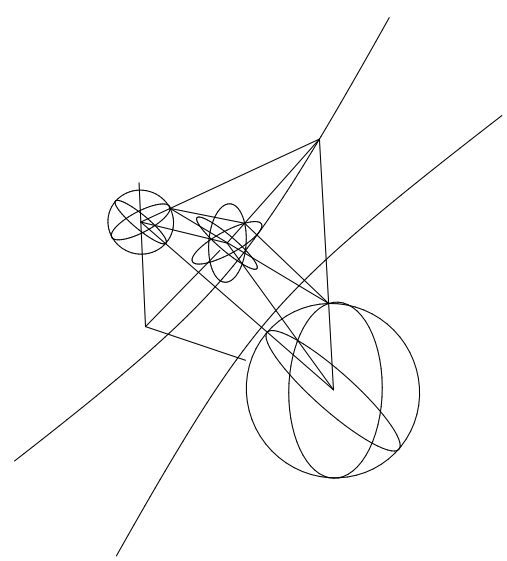

Figure 4. The conflict set of three spheres

If the three spheres are disjoint there is a component of the conflict set that corresponds to

$$
\left|x-p_{1}\right|-r_{1}=\left|x-p_{2}\right|-r_{2}=\left|x-p_{3}\right|-r_{3} .
$$

By the second lemma it is a conic section. This conic is mirrored onto itself through the plane spanned by the $p_{1}=\left(b_{1}, 0,0\right), p_{2}$ and $p_{3}$. This plane is

$$
\frac{x_{1}}{b_{1}}+\frac{x_{2}}{b_{2}}+\frac{x_{3}}{b_{3}}=1
$$

If we denote by $u$ the intersection of the plane (23) with the hyperbola, the parametrization we can use for this component of the conflict set is

$$
t \stackrel{\gamma}{\rightarrow} u+v(\cosh t-1)+w \sinh t .
$$

The vectors $v$ and $w$ determine the plane in which this component of the conflict set lies. With all these remarks it is possible to obtain a parametrization of the conflict set in $\mathbb{R}^{3}$. It is plotted in Figure 4. It can also be verified that the curvature of the conflict set here agrees with the curvature obtained in Theorem 3.1 . 
6. Concluding remarks. We do not doubt that other formulas-possibly more practical ones - can be found. The field of differential geometry is after all fairly replete with all kinds of formulas, the one more suited for actual computation than the other. Doubtless something more is to be discovered here. Let us indicate a few possible generalizations without further proof.

In the example of the previous section we cannot generalize Lemma 5.1 to four surfaces in $\mathbb{R}^{4}$. We can however generalize Lemma 5.2. If we have $l$ spheres in $\mathbb{R}^{n}$ in sufficiently general position, that is $\pm r_{i} \pm r_{j} \neq\left|p_{i}-p_{j}\right|$, then every component of their conflict set is a conic section.

The formula for the normal in (9) has an obvious generalization.

$$
n_{c}=\sum_{i=1}^{l}(-1)^{(i+1)(l+1)} \kappa_{i} e \underset{j=1}{l-2}\left(n_{q(i, j, l)}-n_{q(i+1, j, l)}\right) .
$$

It follows directly from (4). This is the basis for a curvature formula for $n$ surfaces in $\mathbb{R}^{n}$. However calculations tend to get messy and we refrain from carrying out the details.

\section{References}

[GG73] M. Golubitsky, V. Guillemin, Stable Mappings and their Singularities, Graduate Texts in Math. 14, Springer, New York, 1973.

[vM] M. van Manen, The geometry of conflict sets, Dissertation, Univ. Utrecht, 2003.

[Mat68] J. Mather, Stability of $C^{\infty}$ mappings. III. Finitely determined mapgerms, Inst. Hautes Études Sci. Publ. Math. 35 (1968), 279-308.

[Mil63] J. Milnor, Morse Theory, Ann. of Math. Studies 51, Princeton Univ. Press, Princeton, 1963.

[Mon86] James A. Montaldi, On contact between submanifolds, Michigan Math. J. 33 (1986), 195-199.

[Sie99] D. Siersma, Properties of conflict sets in the plane, in: Geometry and Topology of Caustics - Caustics '98, Banach Center Publ. 50, Warszawa, 1999, 267-276.

[Spi] M. Spivak, A Comprehensive Introduction to Differential Geometry, Vol. II, second ed., Publish or Perish Inc., Wilmington, 1979.

[SSG99] J. Sotomayor, D. Siersma, R. Garcia, Curvatures of conflict surfaces in Euclidean 3-space, in: Geometry and Topology of Caustics - Caustics '98, Banach Center Publ. 50, Warszawa, 1999, 277-285. 